\title{
Existence and uniqueness results for fractional differential equations with uncertainty
}

\author{
S Salahshour ${ }^{1 *}$, T Allahviranloo² $^{2}$ S Abbasbandy² and D Baleanu ${ }^{3,4}$
}

\section{"Correspondence:}

soheilsalahshour@yahoo.com

'Young Researchers Club,

Mobarakeh Branch, Islamic Azad University, Mobarakeh, Iran

Full list of author information is

available at the end of the article

\begin{abstract}
In this paper, we study the existence, uniqueness and approximate solutions of fuzzy fractional differential equations (FFDEs) under Caputo's H-differentiability. To this end, the concept of Riemann-Liouville's H-differentiability is introduced, and subsequently, the Caputo's H-differentiability is proposed. Moreover, the related fuzzy Volterra integral forms of FFDEs are obtained which are applied to construct two converge consequences of fuzzy-valued functions as approximated solutions of FFDEs.
\end{abstract}

Keywords: fuzzy fractional differential equations (FFDEs); Riemann-Liouville's H-differentiability; Caputo's H-differentiability

\section{Introduction}

Recently, the theory of fractional differential equations has gained considerable popularity and importance, and as a result, several research papers and monographs have been published in this field (see, for example, [1-11] and the references therein).

As it is known, often the modeling of natural phenomena states using mathematical tools (mathematical arithmetic, mathematical logics and etc.). However, obtaining a deterministic model of such problems is not easy and always has some error and vagueness. So, investigating a popular way to interpret such vagueness is important. Since 1965 with Zadeh's well-known paper on introducing fuzzy sets [12], the applications of a fuzzy concept have appeared more visibly instead of deterministic-stochastic cases. As a result, we need some extensions of mathematical models (e.g., in this contribution, we investigate fractional differential equation) to fuzzy field such that these extensions have natural relationship between crisp and fuzzy cases and even have a natural relation between fuzzy fractional and fuzzy non-fractional cases.

We note that recently Agarwal et al. [13] have proposed the concept of solutions for fractional differential equations with uncertainty. They have considered the RiemannLiouville's differentiability with a fuzzy initial condition to solve FFDEs. However, the differentiability of fuzzy-valued functions in the fractional case was not discussed.

Hence, we will extend the definitions of generalized H-differentiability [14] to the fractional case. To this end, we shall introduce the definition of Riemann-Liouville's Hdifferentiability and Caputo's H-differentiability which are better known than the other types of differentiability in crisp sense [2, 3]. Moreover, we will indicate some properties 
of the mentioned fractional H-differentiability. Consequently, we derive an equivalent integral form of FFDE which allows us to investigate the approximate solutions (for each type of fractional $\mathrm{H}$-differentiability) convergence to the two unique solutions. Note that some related subjects have been discussed in $[15,16]$.

This paper is organized as follows. In Section 2, we review briefly some basic concepts. In Sections 3 and 4, the Riemann-Liouville's H-differentiability and the Caputo's $\mathrm{H}$-differentiability are presented for fuzzy-valued functions, respectively. In Section 5, the approximate solutions of fuzzy fractional differential equations under Caputo's Hdifferentiability are obtained using related equivalent integral forms of original FFDE. Finally, this contribution ends with conclusions.

\section{Basic concepts}

The basic definition of fuzzy numbers is mentioned in [17-19]. We denote the set of all real numbers by $\mathbb{R}$. A fuzzy number represents a mapping $u: \mathbb{R} \rightarrow[0,1]$ fulfilling the following properties:

(a) $u$ is upper semi-continuous,

(b) $u$ is fuzzy convex, i.e., $u(\lambda x+(1-\lambda) y) \geq \min \{u(x), u(y)\}$ for all $x, y \in \mathbb{R}, \lambda \in[0,1]$,

(c) $u$ is normal, i.e., $\exists x_{0} \in \mathbb{R}$ for which $u\left(x_{0}\right)=1$,

(d) $\operatorname{supp} u=\{x \in \mathbb{R} \mid u(x)>0\}$ is the support of the $u$, and its closure $\operatorname{cl}(\operatorname{supp} u)$ is compact.

Let $\mathbb{E}$ be the set of all fuzzy numbers on $\mathbb{R}$. The $r$-level set of a fuzzy number $u \in \mathbb{E}, 0 \leq$ $r \leq 1$, denoted by $[u]_{r}$, is defined as

$$
[u]_{r}= \begin{cases}\{x \in \mathbb{R} \mid u(x) \geq r\} & \text { if } 0<r \leq 1 \\ \operatorname{cl}(\operatorname{supp} u) & \text { if } r=0 .\end{cases}
$$

We notice that the $r$-level set of a fuzzy number is a closed and bounded interval $[\underline{u}(r), \bar{u}(r)]$, where $\underline{u}(r)$ is the left-hand endpoint of $[u]_{r}$ and $\bar{u}(r)$ represents the right-hand endpoint of $[u]_{r}$.

An equivalent parametric definition was presented in [20] as

Definition 2.1 A fuzzy number $u$ in a parametric form is a pair $(\underline{u}, \bar{u})$ of functions $\underline{u}(r)$, $\bar{u}(r), 0 \leq r \leq 1$, which satisfy the following requirements:

1. $\underline{u}(r)$ is a bounded non-decreasing left continuous function in $(0,1]$, and right continuous at 0 ,

2. $\bar{u}(r)$ is a bounded non-increasing left continuous function in $(0,1]$, and right continuous at 0 ,

3. $\underline{u}(r) \leq \bar{u}(r), 0 \leq r \leq 1$.

The Hausdorff distance between fuzzy numbers is given by $d: \mathbb{E} \times \mathbb{E} \longrightarrow[0, \infty]$,

$$
d(u, v)=\sup _{r \in[0,1]} \max \{|\underline{u}(r)-\underline{v}(r)|,|\bar{u}(r)-\bar{v}(r)|\}
$$

where $u=(\underline{u}(r), \bar{u}(r)), v=(\underline{v}(r), \bar{v}(r)) \subset \mathbb{R}$ is utilized in [14]. Then, it is easy to see that $d$ is a metric in $\mathbb{E}$ and has the following properties (see, for example, [21]):

(i) $d(u+w, v+w)=d(u, v), \forall u, v, w \in \mathbb{E}$, 
(ii) $d(k u, k v)=|k| d(u, v), \forall k \in \mathbb{R}, u, v \in \mathbb{E}$,

(iii) $d(u+v, w+e) \leq d(u, w)+d(v, e), \forall u, v, w, e \in \mathbb{E}$,

(iv) $(d, \mathbb{E})$ is a complete metric space.

Theorem 2.1 ([22]) Let $f(x)$ be a fuzzy-valued function on $[a, \infty)$ represented by $(f(x ; r)$, $\bar{f}(x ; r))$. For any fixed $r \in[0,1]$, assume $f(x ; r)$ and $\bar{f}(x ; r)$ are Riemann-integrable on $[a, b]$ for every $b \geq a$, and assume there are two positive functions $M(r)$ and $\bar{M}(r)$ such that $\int_{a}^{b}|f(x ; r)| d x \leq \underline{M}(r)$ and $\int_{a}^{b}|\bar{f}(x ; r)| d x \leq \bar{M}(r)$ for every $b \geq a$. Then, $f(x)$ is improper fuzzy Riemann-integrable on $[a, \infty)$ and the improper fuzzy Riemann-integral is a fuzzy number.

Furthermore, we have

$$
\int_{a}^{\infty} f(x ; r) d x=\left[\int_{a}^{\infty} f(x ; r) d x, \int_{a}^{\infty} \bar{f}(x ; r) d x\right] .
$$

It is known that the H-derivative (differentiability in the sense of Hukuhara) for fuzzy mappings was initially introduced by Puri and Ralescu in [23], and it is based in the Hdifference of sets as follows.

Definition 2.2 Let $x, y \in \mathbb{E}$. If there exists $z \in \mathbb{E}$ such that $x=y+z$, then $z$ is called the $\mathrm{H}$-difference of $\mathrm{x}$ and $\mathrm{y}$, and it is denoted by $x \ominus y$.

We stress the fact that in this manuscript, the sign " $\ominus$ " always stands for $\mathrm{H}$-difference, and also note that $x \ominus y \neq x+(-1) y$.

Let us recall the definition of strongly generalized differentiability introduced in [14].

Definition 2.3 Let $f:(a, b) \rightarrow E$ and $x_{0} \in(a, b)$. We say that $f$ is a strongly generalized differential at $x_{0}$, if there exists an element $f^{\prime}\left(x_{0}\right) \in E$ such that

(i) for all $h>0$ sufficiently small, $\exists f\left(x_{0}+h\right) \ominus f\left(x_{0}\right), \exists f\left(x_{0}\right) \ominus h f\left(x_{0}-h\right)$ and the limits (in the metric $d$ )

$$
\lim _{h \searrow 0} \frac{f\left(x_{0}+h\right) \ominus f\left(x_{0}\right)}{h}=\lim _{h \searrow 0} \frac{f\left(x_{0}\right) \ominus f\left(x_{0}-h\right)}{h}=f^{\prime}\left(x_{0}\right)
$$

or

(ii) for all $h>0$ sufficiently small, $\exists f\left(x_{0}\right) \ominus f\left(x_{0}+h\right), \exists f\left(x_{0}-h\right) \ominus f\left(x_{0}\right)$ and the limits (in the metric $d$ )

$$
\lim _{h \searrow 0} \frac{f\left(x_{0}\right) \ominus f\left(x_{0}+h\right)}{-h}=\lim _{h \searrow 0} \frac{f\left(x_{0}-h\right) \ominus f\left(x_{0}\right)}{-h}=f^{\prime}\left(x_{0}\right)
$$

or

(iii) for all $h>0$ sufficiently small, $\exists f\left(x_{0}+h\right) \ominus f\left(x_{0}\right), \exists f\left(x_{0}-h\right) \ominus f\left(x_{0}\right)$ and the limits (in the metric $d$ )

$$
\lim _{h \searrow 0} \frac{f\left(x_{0}+h\right) \ominus f\left(x_{0}\right)}{h}=\lim _{h \searrow 0} \frac{f\left(x_{0}-h\right) \ominus f\left(x_{0}\right)}{-h}=f^{\prime}\left(x_{0}\right)
$$


(iv) for all $h>0$ sufficiently small, $\exists f\left(x_{0}\right) \ominus f\left(x_{0}+h\right), \exists f\left(x_{0}\right) \ominus f\left(x_{0}-h\right)$ and the limits (in the metric $d$ )

$$
\lim _{h \searrow 0} \frac{f\left(x_{0}\right) \ominus f\left(x_{0}+h\right)}{-h}=\lim _{h \searrow 0} \frac{f\left(x_{0}\right) \ominus f\left(x_{0}-h\right)}{h}=f^{\prime}\left(x_{0}\right) .
$$

Now, we state the definition of a fractional derivative of a real-valued function in the Caputo sense as follows $(C[a, b]$ is a space of real-valued functions which are continuous on $[a, b]$ and $L[a, b]$ is the set of all real-valued measurable functions on $[a, b])$ :

Definition 2.4 Let $f \in C[a, b] \cap L[a, b], f^{\prime}$ is integrable, then the Caputo derivative of $f$ for $0<\beta<1$ and $x \in[a, b]$ is denoted by $\left({ }^{C} D_{a^{+}}^{\beta} f\right)(x) \in R$ and defined by

$$
\left({ }^{C} D_{a^{+}}^{\beta} f\right)(x)=\frac{1}{\Gamma(1-\beta)} \int_{a}^{x}(x-t)^{-\beta} f^{\prime}(t) d t .
$$

\section{Riemann-Liouville's H-differentiability}

In the following, we introduce the definitions of fuzzy Riemann-Liouville integrals and derivatives. We are going to produce such definitions and statements similar to the nonfractional fuzzy cases [14].

Firstly, we define some notations which are used throughout the manuscript.

- $L^{\mathbb{E}}(a, b)$, is the set of all fuzzy-valued measurable functions $f$ on $[a, b]$.

- $C^{\mathbb{E}}[a, b]$ is the space of fuzzy-valued functions which are continuous on $[a, b]$.

- $A C^{\mathbb{E}}[a, b]$ denotes the set of all fuzzy-valued functions which are absolutely continuous.

Notice that one can easily see why we choose such spaces for this contribution in [3] and references therein.

The next step is to define the fuzzy Riemann-Liouville integral of fuzzy-valued function as

Definition 3.1 Let $f: L^{\mathbb{E}} \cap C^{\mathbb{E}}$, the Riemann-Liouville integral of fuzzy-valued function $f$ is as follows:

$$
\left(I_{a+}^{\beta} f\right)(x)=\frac{1}{\Gamma(\beta)} \int_{a}^{x} \frac{f(t) d t}{(x-t)^{1-\beta}}, \quad x>a .
$$

Since, $f(x ; r)=[f(x ; r), \bar{f}(x ; r)]$, for all $0 \leq r \leq 1$, then we can indicate the fuzzy RiemannLiouville integral of fuzzy-valued function $f$ based on the lower and upper functions as follows.

Definition 3.2 Let $f: L^{\mathbb{E}} \cap C^{\mathbb{E}}$, Riemann-Liouville integral of fuzzy-valued function $f$ in the parametric form is

$$
\left(I_{a+}^{\beta} f\right)(x ; r)=\left[\frac{1}{\Gamma(\beta)} \int_{a}^{x} \frac{f(t ; r) d t}{(x-t)^{1-\beta}}, \frac{1}{\Gamma(\beta)} \int_{a}^{x} \frac{\bar{f}(t ; r) d t}{(x-t)^{1-\beta}}\right], \quad x>a, 0 \leq r \leq 1 .
$$

As a result, we define fuzzy Riemann-Liouville fractional differential of order $\beta$ for fuzzy-valued function $f$ as follows. 
Definition 3.3 Let $f: L^{\mathbb{E}} \cap C^{\mathbb{E}}$ and $x_{0} \in(a, b)$ and $\Phi(x)=\frac{1}{\Gamma(1-\beta)} \int_{a}^{x} \frac{f(t) d t}{(x-t)^{\beta}}$. We say that $f(x)$ is fuzzy Riemann-Liouville fractional differentiable of order $0<\beta<1$ at $x_{0}$, if there exists an element $\left({ }^{R L} D_{a^{+}}^{\beta} f\right)\left(x_{0}\right) \in C^{\mathbb{E}}$ such that for all $0 \leq r \leq 1, h>0$

(i) $\left({ }^{R L} D_{a^{+}}^{\beta} f\right)\left(x_{0}\right)=\lim _{h \rightarrow 0^{+}} \frac{\Phi\left(x_{0}+h\right) \ominus \Phi\left(x_{0}\right)}{h}=\lim _{h \rightarrow 0^{+}} \frac{\Phi\left(x_{0}\right) \ominus \Phi\left(x_{0}-h\right)}{h}$,

or

(ii) $\quad\left({ }^{R L} D_{a^{+}}^{\beta} f\right)\left(x_{0}\right)=\lim _{h \rightarrow 0^{+}} \frac{\Phi\left(x_{0}\right) \ominus \Phi\left(x_{0}+h\right)}{-h}=\lim _{h \rightarrow 0^{+}} \frac{\Phi\left(x_{0}-h\right) \ominus \Phi\left(x_{0}\right)}{-h}$.

For the sake of simplicity, we say that a fuzzy-valued function $f$ is ${ }^{R L}[(\mathrm{i})-\beta]$-differentiable if it is differentiable as in the Definition 3.3 case (i), and is ${ }^{R L}[(\mathrm{ii})-\beta]$-differentiable if it is differentiable as in the Definition 3.3 case (ii).

Theorem 3.1 Let $f: L^{\mathbb{E}} \cap C^{\mathbb{E}}$ and $x_{0} \in(a, b), 0<\beta<1$ such that for all $0 \leq r \leq 1$,

(i) iff is ${ }^{R L}[(i)-\beta]$-differentiable, then $f$ and $\bar{f}$ are Riemann-Liouville differentiable functions and

$$
\left({ }^{R L} D_{a^{+}}^{\beta} f\right)\left(x_{0} ; r\right)=\left[{ }^{R L} D_{a^{+}}^{\beta} f\left(x_{0}, r\right),{ }^{R L} D_{a^{+}}^{\beta} \bar{f}\left(x_{0}, r\right)\right] .
$$

(ii) iff is ${ }^{R L}[(i i)-\beta]$-differentiable, then $f$ and $\bar{f}$ are Riemann-Liouville differentiable functions and

$$
\left(D_{a^{+}}^{\beta} f\right)\left(x_{0} ; r\right)=\left[{ }^{R L} D_{a^{+}}^{\beta} \bar{f}\left(x_{0} ; r\right),{ }^{R L} D_{a^{+}}^{\beta} f\left(x_{0}, r\right)\right]
$$

where

$$
{ }^{R L} D_{a^{+}}^{\beta} f\left(x_{0} ; r\right)=\left[\frac{1}{\Gamma(1-\beta)} \frac{d}{d x} \int_{a}^{x} \frac{\bar{f}(t ; r) d t}{(x-t)^{\beta}}\right]_{x=x_{0}},
$$

and

$$
{ }^{R L} D_{a^{+}}^{\beta} \bar{f}\left(x_{0} ; r\right)=\left[\frac{1}{\Gamma(1-\beta)} \frac{d}{d x} \int_{a}^{x} \frac{\bar{f}(t, r) d t}{(x-t)^{1-\beta}}\right]_{x=x_{0}} .
$$

Proof It is easy to prove by using Definition 3.2 and 3.3.

\section{Caputo's H-differentiability}

Now, we present the definitions and some properties of fractional Caputo's H-differentiability. Let $[a, b]$ be a finite interval of the real line $\mathbb{R}$, and let ${ }^{R L} D_{a^{+}}^{\beta}$ be the Riemann-Liouville $\mathrm{H}$-derivative of order $\beta$ introduced by Definition 3.3.

Definition 4.1 Let $f: L^{\mathbb{E}} \cap C^{\mathbb{E}}$ be a fuzzy-valued function. Then $f$ is said to be Caputo's H-differentiable at $x$ when

$$
\left({ }^{C} D_{a^{+}}^{\beta} f\right)(x)=\frac{1}{\Gamma(1-\beta)} \int_{a}^{x}(x-t)^{-\beta} f^{\prime}(t) d t .
$$


Also, we adopt the same procedure to present Caputo's H-differentiability, we say $f$ is ${ }^{C}[(\mathrm{i})-\beta]$-differentiable if Eq. (9) holds while $f$ is (i)-differentiable, and $f$ is ${ }^{C}[(i i)-\beta]$ differentiable if Eq. (9) holds while $f$ is (ii)-differentiable.

Theorem 4.1 Let $0<\beta<1$ and $f \in A C^{\mathbb{E}}[a, b]$, then the fuzzy Caputo fractional derivative exists almost everywhere on $(a, b)$ and for all $0 \leq r \leq 1$ we have

$$
\begin{aligned}
\left({ }^{C} D_{a^{+}}^{\beta} f\right)(x ; r) & =\left[\frac{1}{\Gamma(1-\beta)} \int_{a}^{x} \frac{f^{\prime}(t ; r) d t}{(x-t)^{\beta}}, \frac{1}{\Gamma(1-\beta)} \int_{a}^{x} \frac{\bar{f}^{\prime}(t ; r) d t}{(x-t)^{\beta}}\right] \\
& =\left[\left(I_{a^{+}}^{1-\beta} D f\right)(x ; r),\left(I_{a^{+}}^{1-\beta} D \bar{f}\right)(x ; r)\right],
\end{aligned}
$$

when $f$ is (i)-differentiable, and

$$
\begin{aligned}
\left({ }^{C} D_{a^{+}}^{\beta} f\right)(x ; r) & =\left[\frac{1}{\Gamma(1-\beta)} \int_{a}^{x} \frac{\bar{f}^{\prime}(t ; r) d t}{(x-t)^{\beta}}, \frac{1}{\Gamma(1-\beta)} \int_{a}^{x} \frac{f^{\prime}(t ; r) d t}{(x-t)^{\beta}}\right] \\
& \left.=\left[\left(I_{a^{+}}^{1-\beta} D \bar{f}\right)(x ; r),\left(I_{a^{+}}^{1-\beta} D f\right]\right)(x ; r)\right],
\end{aligned}
$$

when $f$ is (ii)-differentiable.

Proof It is straightforward.

Theorem 4.2 Let us consider $f \in C^{\mathbb{E}}[a, b]$, then we have the following:

$$
\left(I_{a^{+}}^{\beta C} D_{a^{+}}^{\beta} f\right)(x)=f(x) \ominus f(a), \quad 0<\beta<1,
$$

when $f$ is ${ }^{C}[(i)-\beta]$-differentiable and

$$
\left(I_{a^{+}}^{\beta C} D_{a^{+}}^{\beta} f\right)(x)=-f(a) \ominus(-f(x)), \quad 0<\beta<1,
$$

when $f$ is ${ }^{C}[(i i)-\beta]$-differentiable.

Proof Firstly, we recall that $[2,3]$

$$
\begin{aligned}
& \left(I_{a^{+}}^{\beta}{ }^{C} D_{a^{+}}^{\beta} f\right)(x ; r)=f_{-}^{f}(x ; r)-\underset{-}{f}(a ; r), \\
& \left(I_{a^{+}}^{\beta}{ }^{C} D_{a^{+}}^{\beta} \bar{f}\right)(x)=\bar{f}(x ; r)-\bar{f}(a ; r),
\end{aligned}
$$

for the real-valued functions $f$ and $\bar{f}$. So, in the case of ${ }^{C}[(i)-\beta]$-differentiability, we have

$$
\begin{aligned}
\left(I_{a^{+}}^{\beta C} D^{C}{ }_{a^{+}}^{\beta} f\right)(x ; r) & =\left[\left(I_{a^{+}}^{\beta C} D_{a^{+}}^{\beta} f\right)(x ; r),\left(I_{a^{+}}^{\beta C} D_{a^{+}}^{\beta} \bar{f}\right)(x)\right] \\
& =[\underline{f}(x ; r)-\underline{f}(a ; r), \bar{f}(x ; r)-\bar{f}(a ; r)] .
\end{aligned}
$$

Also, for case ${ }^{C}[(\mathrm{ii})-\beta]$-differentiability, we have

$$
\begin{aligned}
\left(I_{a^{+}}^{\beta C} D^{\beta}{ }^{+} f\right)(x ; r) & =\left[\left(I_{a^{+}}^{\beta C} D_{a a^{+}}^{\beta} \bar{f}\right)(x ; r),\left(I_{a^{+}}^{\beta C} D_{a^{+}}^{\beta} f\right)(x ; r)\right] \\
& =[\bar{f}(x ; r)-\bar{f}(a ; r), \underline{f}(x ; r)-f(a ; r)],
\end{aligned}
$$

which completes the proof. 


\section{Approximate solutions}

Now, we investigate the approximate solutions of fuzzy fractional differential equations under Caputo's H-differentiability by using a corresponding equivalent Volterra integral form of original FFDE.

Firstly, we propose the following fuzzy fractional differential equation of order $0<\beta<1$ with the fuzzy initial condition:

$$
\left\{\begin{array}{l}
\left({ }^{C} D_{a^{+}}^{\beta} y\right)(x)=f(x, y(x)), \\
y(a) \in \mathbb{E} .
\end{array}\right.
$$

Lemma 5.1 ([24]) Assume that $g \in C[\Omega, \mathbb{R}]$ where $\Omega$ is an open set, included ordered pairs $(x, u)$ belong to $\mathbb{R}^{2}$, and $\left(x_{0}, u_{0}\right) \in \Omega$. Suppose that $\left[x_{0}, x_{0}+\eta\right]$ is the largest interval of existence of the maximal solution $r(x)$ of $\left({ }^{c} D_{a^{+}}^{\beta} u\right)(x)=g(x, u), u_{0}(x)=u_{0}$. Let $\left[x_{0}, x_{1}\right]$ be a compact interval of $\left[x_{0}, x_{0}+\eta\right]$. Then, there is an $\epsilon_{0}>0$ such that for $0<\epsilon<\epsilon_{0}$, the maximal solution $r(x, \epsilon)$ of $\left({ }^{c} D_{a^{+}}^{\beta} u\right)(x)=g(x, u)+\epsilon, u_{0}(x)=u_{0}+\epsilon$ exists on $\left[x_{0}, x_{1}\right]$ and $\lim _{\epsilon \rightarrow \infty} r(x, \epsilon)=r(x)$ uniformly on $\left[x_{0}, x_{1}\right]$.

Lemma $5.2([24])$ Assume that $m:\left[x_{0}, x_{0}+\eta\right] \longrightarrow[0, \infty]$ is locally Hölder continuous, $g \in C\left[\left[x_{0}, x_{0}+\eta\right] \times[0, \infty],[0, \infty]\right]$ and for $x_{0} \leq x \leq x_{0}+\eta$,

$$
D^{\beta} m(x) \leq g(x, m(x))
$$

Let $r(x)$ be the maximal solution of

$$
D^{\beta} u=g(x, u), \quad u_{0} \geq 0,
$$

existing on $\left[x_{0}, x_{0}+\eta\right]$. Then, we have

$$
m(x) \leq r(x), \quad x_{0} \leq x \leq x_{0}+\eta
$$

The following lemma transforms the fuzzy fractional differential equations to the corresponding fuzzy Volterra integral equations.

Lemma 5.3 Let $0<\beta<1$ and $a \in \mathbb{R}$, the fuzzy fractional differential equation (12) is equivalent to one of the following integral equations:

$$
y(x)=y(a)+\frac{1}{\Gamma(\beta)} \int_{a}^{x} \frac{f(t, y(t)) d t}{(x-t)^{1-\beta}}, \quad x \in[a, b],
$$

if $y$ is ${ }^{C}[(i)-\beta]$-differentiable, and

$$
y(x)=y(a) \ominus \frac{-1}{\Gamma(\beta)} \int_{a}^{x} \frac{f(t, y(t)) d t}{(x-t)^{1-\beta}}, \quad x \in[a, b],
$$

if $y$ is ${ }^{C}[(i i)-\beta]$-differentiable, provided that the H-difference exists.

Here the equivalence between two equations means that any solution of an equation is a solution for the other one too. 
Proof It is obtained immediately by using Theorem 4.2.

Now, we extend Theorem 22 in [14] and Theorem 4.1 in [25] for fuzzy fractional differential equations under ${ }^{C}[(\mathrm{i})-\beta]$ - and ${ }^{C}[(\mathrm{ii})-\beta]$-differentiability.

Theorem 5.1 Suppose that the following conditions hold: (a) Let $R_{0}=\left[x_{0}, x_{0}+p\right] \times \bar{B}\left(y_{0}, q\right)$, $p, q>0, y_{0} \in E$, where $\bar{B}\left(y_{0}, q\right)=\left\{y \in \mathbb{E}: d\left(y, y_{0}\right) \leq q\right\}$ denote a closed ball in $\mathbb{E}$ and let $f: R_{0} \rightarrow \mathbb{E}$ be a continuous function such that $d(\widetilde{0}, f(x, y))=\|f(x, y)\| \leq M \widetilde{(0}$ is fuzzy zero such that it is a singleton) for all $(x, y) \in R_{0}$. (b) Let $g:\left[x_{0}, x_{0}+p\right] \times[0, q] \rightarrow \mathbb{E}$, such that $g(x, 0) \equiv 0$ and $0 \leq g(x, u) \leq M_{1}, \forall x \in\left[x_{0}, x_{0}+p\right], 0 \leq u \leq q$, such that $g(x, u)$ is non-decreasing in $u$ and $g$ is such that the initial-value problem $\left({ }^{C} D_{a^{+}}^{\beta} u\right)(x)=g(x, u(x))$, $u\left(x_{0}\right)=0$ has only the solution $u(x) \equiv 0$ on $\left[x_{0}, x_{0}+p\right]$. (c) We have $d\left(\frac{f(x, y)}{\left(x_{1}-s\right)^{1-\beta}}, \frac{f(x, z)}{\left(x_{2}-s\right)^{1-\beta}}\right) \leq$ $\left|\left(x_{1}-s\right)^{\beta-1}-\left(x_{2}-s\right)^{\beta-1}\right| \cdot g(x, d(y, z)), \forall(x, y),(x, z) \in R_{0}, x_{1}, x_{2}, s \in\left[x_{0}, x_{0}+p\right]$ and $d(y, z) \leq q$. (d) There exists $d>0$ such that for $x \in\left[x_{0}, x_{0}+d\right]$ the sequence $\widehat{y}_{n}:\left[x_{0}, x_{0}+d\right] \rightarrow \mathbb{E}$ given by $\widehat{y}_{0}(x)=y_{0}, \widehat{y}_{n+1}(x)=y_{0} \ominus \frac{(-1)}{\Gamma(\beta)} \cdot \int_{x_{0}}^{x} \frac{f\left(t, \widehat{y}_{n}\right) d t}{(x-t)^{1-\beta}}$ is defined for any $n \in \mathbb{N}$.

Then, the following FFDE

$$
\left\{\begin{array}{l}
\left({ }^{C} D_{a^{+}}^{\beta} y\right)(x)=f(x, y(x)), \\
y\left(x_{0}\right)=y_{0} \in \mathbb{E}
\end{array}\right.
$$

has two solutions (one ${ }^{C}[(i)-\beta]$-differentiable and the other one ${ }^{C}[($ ii $)-\beta]$-differentiable) $y, \widehat{y}:\left[x_{0}, x_{0}+r\right] \longrightarrow B\left(y_{0}, q\right)$ where $r=\min \left\{p,\left(\frac{q \Gamma(\beta+1)}{M}\right)^{\frac{1}{\beta}},\left(\frac{q \Gamma(\beta+1)}{M_{1}}\right)^{\frac{1}{\beta}}, d\right\}$ and the successive iterations

$$
y_{0}(x)=y_{0}, \quad y_{n+1}(x)=y_{0}+\frac{1}{\Gamma(\beta)} \int_{x_{0}}^{x} \frac{f\left(t, y_{n}(t)\right)}{(x-t)^{1-\beta}} d t, \quad 0<\beta \leq 1,
$$

and

$$
\widehat{y}_{0}(x)=y_{0}, \quad \widehat{y}_{n+1}(x)=y_{0} \ominus \frac{(-1)}{\Gamma(\beta)} \int_{x_{0}}^{x} \frac{f\left(t, \widehat{y}_{n}(t)\right)}{(x-t)^{1-\beta}} d t, \quad 0<\beta \leq 1,
$$

converge to these two solutions respectively provided that the H-difference exists.

Proof Without loss of generality, we prove for case ${ }^{C}[(\mathrm{i})-\beta]$-differentiability. The proof of the second case is completely similar to previous one and so it is omitted.

For $x_{0} \leq x_{1} \leq x_{2} \leq x_{0}+r$ and $M_{2}=\max \left\{M, M_{1}\right\}$, we have

$$
\begin{aligned}
d\left(y_{1}\left(x_{1}\right), y_{1}\left(x_{2}\right)\right) \leq & d\left(y_{0}\left(x_{1}\right), y_{0}\left(x_{2}\right)\right)+\frac{1}{\Gamma(\beta)} \int_{x_{0}}^{x_{1}} d\left(\frac{f\left(s, y_{n-1}(s)\right)}{\left(x_{1}-s\right)^{1-\beta}}, \frac{f\left(s, y_{n-1}(s)\right)}{\left(x_{2}-s\right)^{1-\beta}}\right) d s \\
& +\frac{1}{\Gamma(\beta)} \int_{x_{1}}^{x_{2}} d\left(\frac{f\left(s, y_{n-1}(s)\right)}{\left(x_{2}-s\right)^{1-\beta}}, \widetilde{0}\right) d s \\
\leq & d\left(y_{0}\left(x_{1}\right), y_{0}\left(x_{2}\right)\right)+\frac{M_{2}}{\Gamma(\beta)} \int_{x_{0}}^{x_{1}}\left|\left(x_{1}-s\right)^{\beta-1}-\left(x_{2}-s\right)^{\beta-1}\right| d s \\
& +\frac{M_{2}}{\Gamma(\beta)} \int_{x_{1}}^{x_{2}} \frac{d s}{\left(x_{2}-s\right)^{1-\beta}} \\
\leq & d\left(y_{0}\left(x_{1}\right), y_{0}\left(x_{2}\right)\right)+\frac{M_{2}}{\Gamma(\beta+1)}\left[\left|x_{1}-x_{2}\right|^{\beta}-\left|x_{1}^{\beta}-x_{2}^{\beta}\right|\right]
\end{aligned}
$$




$$
\begin{aligned}
& +\frac{M_{2}}{\Gamma(\beta+1)}\left|x_{1}-x_{2}\right|^{\beta} \\
\leq & d\left(y_{0}\left(x_{1}\right), y_{0}\left(x_{2}\right)\right)+\frac{M_{2}}{\Gamma(\beta+1)}\left[2\left|x_{1}-x_{2}\right|^{\beta}-\left|x_{1}^{\beta}-x_{2}^{\beta}\right|\right] \\
\leq & d\left(y_{0}\left(x_{1}\right), y_{0}\left(x_{2}\right)\right)+\frac{2 M_{2}}{\Gamma(\beta+1)}\left|x_{1}-x_{2}\right|^{\beta} \\
< & \epsilon
\end{aligned}
$$

provided $\left|x_{1}-x_{2}\right|<\delta$, where $\delta=\left[\frac{\epsilon \Gamma(\beta+1)}{4 M_{2}}\right]^{\frac{1}{\beta}}$ and under assumption $d\left(y_{0}\left(x_{1}\right), y_{0}\left(x_{2}\right)\right)<\frac{\epsilon}{2}$, proving that $y_{1}(x)$ is continuous on $\left[x_{0}, x_{0}+r\right]$.

Similarly,

$$
\begin{aligned}
d\left(y_{1}(x), y_{0}(x)\right) & \leq \frac{1}{\Gamma(\beta)} \int_{x_{0}}^{x}(x-s)^{\beta-1} d\left(f\left(s, y_{0}\right), \widetilde{0}\right) d s \\
& \leq \frac{M_{2}\left(x-x_{0}\right)^{\beta}}{\Gamma(\beta+1)} \leq \frac{M_{2} r^{\beta}}{\Gamma(\beta+1)} \leq q .
\end{aligned}
$$

Thus, it is easily obtained that the successive approximations are continuous and satisfy the following relation:

$$
d\left(y_{n+1}(x), y_{0}(x)\right) \leq q, \quad \forall x \in\left[x_{0}, x_{0}+r\right], n=0,1,2,3, \ldots
$$

Hence, $y_{n+1} \in C\left[\left[x_{0}, x_{0}+r\right], B\left(x_{0}, q\right)\right]$ and

$$
\left({ }^{C} D_{a^{+}}^{\beta} y_{n+1}\right)(x)=f\left(x, y_{n}(x)\right), \quad y_{n}\left(x_{0}\right)=y_{0}, n=0,1,2,3, \ldots
$$

Now, we define the following successive approximations for $r=\min \left\{p,\left[\frac{q \Gamma(\beta+1)}{M_{2}}\right]^{\frac{1}{\beta}}\right\}$ :

$$
\begin{aligned}
& u_{0}(x)=\frac{M_{2}\left(x-x_{0}\right)^{\beta}}{\Gamma(\beta+1)}, \quad x \in\left[x_{0}, x_{0}+r\right], \\
& u_{n+1}(x)=\frac{1}{\Gamma(\beta)} \int_{x_{0}}^{x}(x-s)^{\beta-1} g\left(s, u_{n}(s)\right) d s, \quad x \in\left[x_{0}, x_{0}+r\right] .
\end{aligned}
$$

Then, we get immediately

$$
\begin{aligned}
u_{1}(x) & =\frac{1}{\Gamma(\beta)} \int_{x_{0}}^{x}(x-s)^{\beta-1} g\left(s, u_{0}(s)\right) d s \\
& \leq M_{1}\left(x-x_{0}\right) \leq u_{0}(x) \leq q, \quad \forall x \in\left[x_{0}, x_{0}+r\right] .
\end{aligned}
$$

Hence, by the inductive method and in view that $g(x, u)$ is nondecreasing on $u$, we get

$$
0 \leq u_{n+1}(x) \leq u_{n}(x) \leq q, \quad \forall x \in\left[x_{0}, x_{0}+r\right], n=0,1,2,3, \ldots
$$

Moreover, $\left|\left({ }^{C} D_{a^{+}}^{\beta} u_{n+1}\right)(x)\right|=\left|g\left(x, u_{n}(x)\right)\right| \leq M_{1}$; and therefore, we can conclude by AscoliArzela theorem and the monotonicity of the sequence $\left\{u_{n}(x)\right\}$ that $\lim _{n \rightarrow \infty} u_{n}(x)=u(x)$ uniformly on $\left[x_{0}, x_{0}+r\right]$ and

$$
u(x)=\frac{1}{\Gamma(\beta)} \int_{x_{0}}^{x} \frac{g(s, u(s)) d s}{(x-s)^{1-\beta}} .
$$


Thus, $u \in C\left[\left[x_{0}, x_{0}+r\right],[0, q]\right]$ and $u(x)$ is the solution of the initial value problem (20).

From assumption (b), we get $u(x) \equiv 0$. Moreover, we have

$$
\begin{aligned}
d\left(y_{1}(x), y_{0}(x)\right) & =d\left(\int_{x_{0}}^{x} \frac{f\left(s, y_{0}(s)\right) d s}{(x-s)^{1-\beta}}, \tilde{0}\right) \\
& \leq \int_{x_{0}}^{x} d\left(\frac{f\left(s, y_{0}(s)\right) d s}{(x-s)^{1-\beta}}, \tilde{0}\right) \\
& \leq \frac{M_{2}\left(x-x_{0}\right)^{\beta}}{\Gamma(\beta+1)}=u_{0}(x) .
\end{aligned}
$$

Suppose that $d\left(y_{k}(x), y_{k-1}(x)\right) \leq u_{k-1}(x)$, then using assumption (c), we have

$$
\begin{aligned}
d\left(y_{k+1}(x), y_{k}(x)\right) & =\frac{1}{\Gamma(\beta)} \cdot d\left(\int_{x_{0}}^{x} \frac{f\left(s, y_{k}(s) d s\right.}{(x-s)^{1-\beta}}, \frac{f\left(s, y_{k-1}(s) d s\right.}{(x-s)^{1-\beta}}\right) \\
& \leq \frac{1}{\Gamma(\beta)} \cdot \int_{x_{0}}^{x} d\left(\frac{f\left(s, y_{k}(s)\right.}{(x-s)^{1-\beta}}, \frac{f\left(s, y_{k-1}(s)\right.}{(x-s)^{1-\beta}}\right) d s \\
& \leq \frac{1}{\Gamma(\beta)} \cdot \int_{x_{0}}^{x} \frac{g\left(s, d\left(y_{k}(s), y_{k-1}(s)\right)\right)}{(x-s)^{1-\beta}} d s \\
& \leq \frac{1}{\Gamma(\beta)} \cdot \int_{x_{0}}^{x} \frac{g\left(s, u_{k-1}(s)\right)}{(x-s)^{1-\beta}} d s \\
& =u_{k}(x) .
\end{aligned}
$$

Thus, by the inductive method, we know

$$
d\left(y_{n+1}(x), y_{n}(x)\right) \leq u_{n}(x), \quad \forall x \in\left[x_{0}, x_{0}+r\right], n=0,1,2,3, \ldots
$$

So, we have

$$
\begin{aligned}
d\left(\left({ }^{C} D_{a^{+}}^{\beta} y_{n+1}\right)(x),\left({ }^{C} D_{a^{+}}^{\beta} y_{n}\right)(x)\right) & =d\left(f\left(x, y_{n}(x)\right), f\left(x, y_{n-1}(x)\right)\right) \\
& \leq g\left(x, d\left(y_{n}(x), y_{n-1}(x)\right)\right) \\
& \leq g\left(x, u_{n-1}(x)\right) .
\end{aligned}
$$

Assume $m \geq n$, then one can easily obtain

$$
\begin{aligned}
{ }^{C} D_{a^{+}}^{+\beta}\left(d\left(y_{n}(x), y_{m}(x)\right)\right) & \leq d\left(\left({ }^{C} D_{a^{+}}^{\beta} y_{n}\right)(x),\left({ }^{C} D_{a^{+}}^{\beta} y_{m}\right)(x)\right) \\
& \leq g\left(x, u_{n-1}(x)\right)+g\left(x, u_{m-1}(x)\right)+g\left(x, d\left(y_{n}(x), y_{m}(x)\right)\right) .
\end{aligned}
$$

Since, $u_{n+1} \leq u_{n}(x)$ for all $n$, it follows that:

$$
{ }^{C} D_{a^{+}}^{+\beta}\left(d\left(y_{n}(x), y_{m}(x)\right)\right) \leq 2 g\left(x, u_{n-1}(x)\right)+g\left(x, d\left(y_{n}(x), y_{m}(x)\right)\right),
$$

where ${ }^{C} D_{a^{+}}^{+\beta}$ denotes the corresponding Dini derivative to $D^{+}[24]$.

Since $g\left(x, u_{n-1}(x)\right)$ uniformly converges to 0 , then for arbitrary $\epsilon>0$, there exists a natural number $N$ such that

$$
{ }^{C} D_{a^{+}}^{+\beta}\left(d\left(y_{n}(x), y_{m}(x)\right)\right) \leq g\left(x, d\left(y_{n}(x), y_{m}(x)\right)\right)+\epsilon, \quad \forall m \geq n>N
$$


From the fact that $D\left(y_{n}\left(x_{0}\right), y_{m}\left(x_{0}\right)\right)=0$ and by using Lemma 5.2 , we have

$$
d\left(y_{n}(x), y_{m}(x)\right) \leq r(x, \epsilon), \quad \forall x \in\left[x_{0}, x_{0}+r\right], m \geq n>N
$$

where $r(x, \epsilon)$ is the maximal solution to the following initial value problem for each $n$ :

$$
\left({ }^{C} D_{a^{+}}^{\beta} v\right)(x)=g(x, v)+2 g\left(x, u_{n-1}(x)\right), \quad v\left(x_{0}\right)=0 .
$$

Since, as $n \longrightarrow \infty, 2 g\left(x, u_{n-1}(x)\right) \longrightarrow 0$ uniformly on $\left[x_{0}, x_{0}+r\right]$, it follows by Lemma 5.1 that $r_{n}(x) \longrightarrow 0$ uniformly on $\left[x_{0}, x_{0}+r\right]$.

Hence, according to $(22)$ and the fact that $(\mathbb{E}, d)$ is complete, we conclude that there exists a fuzzy-valued function $y:\left[x_{0}, x_{0}+r\right] \longrightarrow \mathbb{E}$ such that $d\left(y_{n}(x), y_{m}(x)\right)$ uniformly converges to 0 as $n \longrightarrow \infty$. Applying (18) and Lemma 5.3 we have $y \in C\left[\left[x_{0}, x_{0}+r\right], B\left(x_{0}, q\right)\right]$ and $y(x)$ is the solution of FFDE (15).

Now, we show the uniqueness of solution of Eq. (15). Let us consider $w(x)$ is another solution of Eq. (15) on $\left[x_{0}, x_{0}+r\right]$. Also, define $m(x)=d(y(x), w(x))$, then $m\left(x_{0}\right)=0$ and

$$
\begin{aligned}
\left({ }^{C} D_{a^{+}}^{+\beta} m\right)(x) & \leq d\left(\left({ }^{C} D_{a^{+}}^{\beta} y\right)(x),\left({ }^{C} D_{a^{+}}^{\beta} w\right)(x)\right) \\
& =d(f(x, y(x)), f(x, w(x))) \\
& \leq g(x, m(x)) .
\end{aligned}
$$

Hence, from Lemma 5.2, we have $d(y(x), w(x)) \leq u(x) \equiv 0$ for all $r \in\left[x_{0}, x_{0}+r\right]$, where $u(x) \equiv 0$ is the maximal solution of

$$
\left({ }^{C} D_{a^{+}}^{\beta} u\right)(x)=g(x, u(x)), \quad u\left(x_{0}\right)=0 .
$$

Therefore, $y(x)=w(x)$, which completes the proof.

The following Peano-type theorem is given to derive solutions of fuzzy fractional differential equations with order $0<\beta<1$ under Caputo's differentiability.

Theorem 5.2 Let $R_{0}=\left[x_{0}, x_{0}+p\right] \times \bar{B}\left(y_{0}, q\right), p, q>0, y_{0} \in E$, where $\bar{B}\left(y_{0}, q\right)=\{y \in \mathbb{E}$ : $d\left(y, y_{0}\right) \leq q$ \} denote a closed ball in $\mathbb{E}$ and let $f: R_{0} \longrightarrow \mathbb{E}$ be a continuous function such that $d(\tilde{0}, f(x, y))=\|f(x, y)\| \leq M$ for all $(x, y) \in R_{0}$ and $f$ satisfies the Lipschitz condition $d\left(\left(x_{1}-s\right)^{\beta-1} f(x, y),\left(x_{2}-s\right)^{\beta-1} f(x, z)\right) \leq\left|\left(x_{1}-s\right)^{\beta-1}-\left(x_{2}-s\right)^{\beta-1}\right| \cdot L \cdot d(y, z) \forall(x, y),(x, z) \in R_{0}$, $x_{1}, x_{2}, s \in\left[x_{0}, x_{0}+p\right]$ and $d(y, z) \leq q$. If there exists $d>0$ such that for $x \in\left[x_{0}, x_{0}+d\right]$ the sequence given by $\widehat{y}_{0}(x)=y_{0}, \widehat{y}_{n+1}(x)=y_{0} \ominus \frac{(-1)}{\Gamma(\beta)} \cdot \int_{x_{0}}^{x} \frac{f\left(t, \widehat{y}_{n}\right)}{(x-t)^{1-\beta}} d t$ is defined for any $n \in \mathbb{N}$. Then, the following fuzzy fractional initial-value problem:

$$
\left\{\begin{array}{l}
\left({ }^{C} D_{a^{+}}^{\beta} y\right)(x)=f(x, y(x)), \\
y\left(x_{0}\right)=y_{0}
\end{array}\right.
$$

has two solutions $y, \widehat{y}:\left[x_{0}, x_{0}+r\right] \longrightarrow B\left(y_{0}, q\right)$ where $r=\min \left\{p,\left(\frac{q \Gamma(\beta+1)}{M}\right)^{\frac{1}{\beta}},\left(\frac{q \Gamma(\beta+1)}{M_{1}}\right)^{\frac{1}{\beta}}, d\right\}$ and the successive iterations in Theorem 5.1 converge to these two solutions.

Proof The proof is obtained immediately by taking in Theorem 5.1, $g(x, u)=L \Delta u$. 


\section{Conclusion}

In this manuscript, we have studied the approximate solutions of fuzzy fractional differential equations under Riemann-Liouville and Caputo's H-differentiability. To this end, some results in each case of differentiability were derived which led to transform the original FFDE to the corresponding fuzzy Volterra integral equations. Then, we have constructed two successive iterations that converge to the solutions of original FFDE under each type of Caputo's H-differentiability.

\section{Competing interests}

The authors declare that they have no competing interests.

\section{Authors' contributions}

SS wrote the first draft, TA and SA corrected and improved it and DB prepared the final version.

\section{Author details}

${ }^{1}$ Young Researchers Club, Mobarakeh Branch, Islamic Azad University, Mobarakeh, Iran. ²Department of Mathematics, Science and Research Branch, Islamic Azad University, Tehran, Iran. ${ }^{3}$ Department of Mathematics and Computer Science, Cakaya University, 06530 Ankara, Turkey. ${ }^{4}$ Institute of Space Sciences, Magurele-Bucharest, Romania.

\section{Acknowledgement}

The authors thank the referee for careful reading and helpful suggestions on the improvement of the manuscript.

Received: 3 May 2012 Accepted: 10 July 2012 Published: 19 July 2012

\section{References}

1. Samko, SG, Kilbas, A, Marichev, Ol: Fractional Integrals Derivatives - Theory and Applications. Gordon and Breach, New York (1993)

2. Podlubny, I: Fractional Differential Equation. Academic Press, San Diego (1999)

3. Kilbas, AA, Srivastava, HM, Trujillo, JJ: Theory and Applications of Fractional Differential Equations. Elsevier, Amsterdam (2006)

4. Miller, KS, Ross, B: An Introduction to the Fractional Calculus and Differential Equations. Wiley, New York (1993)

5. West, BJ, Bologna, M, Grogolini, P: Physics of Fractal Operators. Springer, New York (2003)

6. Magin, RL: Fractional Calculus in Bioengineering. Begell House Publisher, Inc., West Redding (2006)

7. Baleanu, D, Diethelm, K, Scalas, E, Trujillo, Jj: Fractional Calculus Models and Numerical Methods. Series on Complexity, Nonlinearity and Chaos. World Scientific, Singapore (2012)

8. Lakshmikantham, V, Leela, S, Vasundhara Devi, J: Theory of Fractional Dynamic Systems. Cambridge Scientific Pub., Cambridge, UK (2009)

9. Ahmad, B, Nieto, JJ: Existence results for a coupled system of nonlinear fractional differential equations with three-point boundary conditions. Comput. Math. Appl. 58, 1838-1843 (2009)

10. Diethelm, K, Ford, NJ: Analysis of fractional differential equations. J. Math. Anal. Appl. 265, 229-248 (2002)

11. Abbasbandy, S: An approximation solution of a nonlinear equation with Riemann-Liouville's fractional derivatives by He's variational iteration method. J. Comput. Appl. Math. 207, 53-58 (2007)

12. Zadeh, LA: Fuzzy sets. Inf. Control 8, 338-353 (1965)

13. Agarwal, RP, Lakshmikantham, V, Nieto, JJ: On the concept of solution for fractional differential equations with uncertainty. Nonlinear Anal. 72, 2859-2862 (2010)

14. Bede, B, Gal, SG: Generalizations of the differentiability of fuzzy-number-valued functions with applications to fuzzy differential equations. Fuzzy Sets Syst. 151, 581-599 (2005)

15. Allahviranloo, T, Salahshour, S, Abbasbandy, S: Explicit solutions of fractional differential equations with uncertainty. Soft Comput. 16, 297-302 (2012)

16. Salahshour, S, Allahviranloo, T, Abbasbandy, S: Solving fuzzy fractional differential equations by fuzzy Laplace transforms. Commun. Nonlinear Sci. Numer. Simul. 17, 1372-1381 (2012)

17. Diamond, P, Kloeden, PE: Metric Spaces of Fuzzy Sets. World Scientific, Singapore (1994)

18. Dubios, D, Prade, H: Towards fuzzy differential calculus. Fuzzy Sets Syst. 8, 225-233 (1982)

19. Lakshmikantham, V, Mohapatra, RN: Theory of Fuzzy Differential Equations and Applications. Taylor \& Francis, London (2003)

20. Friedman, M, Ming, M, Kandel, A: Numerical solution of fuzzy differential and integral equations. Fuzzy Sets Syst. 106, 35-48 (1999)

21. Puri, ML, Ralescu, D: Fuzzy random variables. J. Math. Anal. Appl. 114, 409-422 (1986)

22. Wu, HC: The improper fuzzy Riemann integral and its numerical integration. Inf. Sci. 111, 109-137 (1999)

23. Puri, ML, Ralescu, D: Differential for fuzzy function. J. Math. Anal. Appl. 91, 552-558 (1983)

24. Lakshmikantham, V, Vasundhara Devi, J: Theory of fractional differential equations in a Banach space. Eur. J. Pure Appl. Math. 1, 38-45 (2008)

25. Wu, C, Song, S, Lee, ES: Approximate solutions, existence, and uniqueness of the Cauchy problem of fuzzy differential equations. J. Math. Anal. Appl. 202, 629-644 (1996) 